\title{
Identification of key genes associated with congenital heart defects in embryos of diabetic mice
}

\author{
$\mathrm{NAN} \mathrm{LIN}^{1 *}, \mathrm{YAN} \mathrm{CAI}^{1 *}, \mathrm{LINLIN} \mathrm{ZHANG}^{2}$ and YAHANG CHEN ${ }^{3}$ \\ ${ }^{1}$ Department of Obstetrics and Gynecology, The Fourth Affiliated Hospital of Harbin Medical University; \\ ${ }^{2}$ Gastroenterology Department, Harbin The First Hospital; ${ }^{3}$ Department of Obstetrics and Gynecology, \\ The Hospital of Heilongjiang, Harbin, Heilongjiang 150001, P.R. China
}

Received March 10, 2017; Accepted September 5, 2017

DOI: $10.3892 / \mathrm{mmr} .2017 .8330$

\begin{abstract}
Maternal diabetes has been reported to be a critical factor for congenital heart defects (CHD) in offspring. The present study aimed to screen the key genes that may be involved in CHD in offspring of diabetic mothers. The present study obtained the gene expression profile of GSE32078, including three embryonic heart tissue samples at embryonic day 13.5 (E13.5), three embryonic heart tissue samples at embryonic day 15.5 (E15.5) from diabetic mice and their respective controls from normal mice. The cut-off criterion of $\mathrm{P}<0.08$ was set to screen differentially expressed genes (DEGs). Their enrichment functions were predicted by Gene Ontology. The enriched pathways were forecasted by Kyoto Encyclopedia of Genes and Genomes and Reactome analysis. Protein-protein interaction (PPI) networks for DEGs were constructed using Cytoscape. The present study identified 869 and 802 DEGs in E13.5 group and E15.5 group, respectively and 182 DEGs were shared by the two developmental stages. The pathway enrichment analysis results revealed that DEGs including intercellular adhesion molecule 1 (Icaml) and H2-M9 were enriched in cell adhesion molecules; DEGs including bone morphogenetic protein receptor type $1 \mathrm{~A}$, transforming growth factor $\beta$ receptor 1 and SMAD specific E3 ubiquitin protein ligase 1 were enriched in the tumor growth factor- $\beta$ signaling pathway. In addition, DEGs including Icaml, Cls and Fc fragment of IgG receptor IIb were enriched in Staphylococcus aureus infection. Furthermore, the shared DEGs including Icam1, nuclear receptor corepressor 1 (Ncorl) and AKT serine/threonine kinase 3 (Akt3) had high connectivity degrees in the PPI network. The shared DEGs including Icaml, Ncorl
\end{abstract}

Correspondence to: Dr Nan Lin, Department of Obstetrics and Gynecology, The Fourth Affiliated Hospital of Harbin Medical University, 37 Yiyuan Street, Harbin, Heilongjiang 150001, P.R. China E-mail: linnan_zll@126.com

*Contributed equally

Key words: congenital heart defects, diabetes, differentially expressed genes, pathway, protein-protein interaction and Akt3 may be important in the cardiogenesis of embryos. These genes may be involved in the development of CHD in the offspring of diabetic mothers.

\section{Introduction}

Diabetes mellitus is a chronic disease characterized by hyperglycemia resulting from defects in insulin secretion, insulin action, or both (1). According to insulin-dependent and non-insulin-dependent, adult diabetes is classified: Type 1 and type 2 diabetes. A genetic defect on insulin was found in type 1 diabetes, whereas insulin resistance is the key metabolic abnormality in type 2 diabetes (2). In addition, maternal diabetes in pregnancy is defined as glucose intolerance or first recognition during pregnancy, who is easy to happen type 2 diabetes postpartum (3). Besides, maternal diabetes in pregnancy is related to an increased risk of congenital heart defects (CHD), macrosomia, miscarriage, and other birth defects in offspring $(4,5)$. CHD was defined as deficits of the structure and function arising from cardiac embryogenesis stage (6). It has been reported that CHD is the major consequences in diabetic embryopathy (7).

An infant born to a diabetic mother has been shown to exhibit axial mesodermal dysplasia spectrum with atrioventricular septal defects (8). Many studies have reported that maternal diabetes altered expression of genes in developmental embryo $(4,9,10)$. Bmp 4, belongs to the TGF- $\beta$ superfamily, is a myocardial signaling molecule which activated epithelial-mesenchymal transition (EMT) during cardiogenesis (11). The expression of $\mathrm{Bmp} 4$ has been reported to be downregulated by the $M s x 1$ that expressed in atrioventricular canal endocardial cells during EMT $(12,13)$. Pax3 is also essential for heart formation and outflow tract development in the mouse embryo (14). Study showed that the downregulation of Bmp4, Msxl and Pax3 could contribute to the pathogenesis of maternal diabetes-induced CHD (15). In addition, it has been reported that hyperglycemia altered the expression of $e N O C$ and $V E G F$ that are involved in the regulation of vasculogenesis (16). Furthermore, a recent study on microarray analysis showed that several genes were altered in embryonic heart tissues from diabetic mother and were closely associated with CHD, such as Smydl, Tscl and Gjal (4). However, the exact pathogenesis of CHD in offspring of diabetic mother is still unknown. 
In this study, we explored the gene expression profiles of embryonic heart tissue samples at embryonic day 13.5 (E13.5) and embryonic day 15.5 (E15.5) from diabetic mice, and their respective controls. The differentially expressed genes (DEGs) were screened in E13.5 and E15.5 groups. Gene Ontology (GO) functions, Kyoto Encyclopedia of Genes and Genomes (KEGG) and Reactome pathway enrichment analyses were then performed to identify the DEGs. We also constructed the protein-protein interaction (PPI) networks for the DEGs and analyzed several important shared genes that were associated with CHD. Our study aimed to identify the critical genes that might be involved in CHD in offspring of diabetic mother and to provide evidence to further clarify the relationship between CHD and diabetes.

\section{Materials and methods}

Affymetrix microarray data. The gene expression profile of GSE32078 was obtained from the study of Vijaya et al (4), which was deposited in Gene Expression Omnibus (GEO) database (http://www.ncbi.nlm.nih.gov/geo/) of National Center of Biotechnology Information (NCBI). A total of 12 samples were available, including three embryonic heart tissue samples at E13.5 and three embryonic heart tissue samples at E15.5 from streptozotocin-induced diabetic Swiss albino mice (8-10 weeks), as well as their respective controls from normal mice. The day when a copulation plug was observed was counted as E0.5. Raw data were collected with the Affymetrix Mouse Genome 430 2.0 Array (Affymetrix, CA, USA). All raw data files were pretreated by RMA method in Affy package (17).

Screening of DEGs. The Linear Models for Microarray Data (LIMMA) package (18) was used to identify DEGs. All genes were tested with $F$-test and the DEGs with the threshold P-value $<0.08$ were screened.

GO andKEGGpathwayenrichmentanalysis.ClusterProfiler, a new ontology-based tool, offers three methods (group $\mathrm{GO}$, enrich $\mathrm{GO}$, enrich KEGG) for genes classification and enrichment analyses $(19,20)$. ClusterProfiler package was used to identify the main biological processes and metabolic pathways in DEGs. Default parameters (organism, mouse; ont, BP; P-value cut-off, 0.05; P-adjust method, none; readable, $\mathrm{T}$ ) were used as the cut-off criteria for $\mathrm{GO}$ function enrichment analysis. And the default parameters (organism, mouse; P-value cut-off, 1; q-value cut-off, 1; readable, 1) were used as the cut-off criteria for KEGG pathway enrichment analysis.

Reactome pathway enrichment analysis. ReactomePA package was used to identify the main biological processes and metabolic pathways in DEGs $(21,22)$. Default parameters (organism, mouse; P-value cut-off, 1; q-value cut-off, 1; minGSSize, 1; readable, 1) were used as the cut-off criteria for Reactome pathway enrichment analysis.

PPI network construction. The Search Tool for the Retrieval of Interacting Genes (STRING) database was used to obtain PPI data. The PPI networks of up- and downregulated DEGs were visualized by Cytoscape (http://cytoscape.org/) that is an open source software for visualizing complex networks and integrating these networks with any type of attribute data (23). The Required Confidence score $>0.4$ was chosen as the threshold.

\section{Results}

Screening of DEGs. The microarray dataset GSE32078 from GEO database was obtained to identify the DEGs in E13.5 and E15.5 hearts of embryos from diabetic mice compared with their respective controls. Totally, 869 DEGs were obtained in E13.5 group when compared with the control group, including 411 up- and 458 downregulated DEGs. Meanwhile, 802 genes were upregulated and 1,295 genes were downregulated in E15.5 group when compared with the control group. Finally, a total of 182 DEGs were shared between E13.5 and E15.5 groups, including 63 up- and 119 downregulated DEGs.

GO function enrichment analysis of DEGs. According to the GO enrichment analysis, we found that the upregulated DEGs in E13.5 group were mainly enriched in developmental process $(\mathrm{P}=2.32 \mathrm{E}-08)$ and the downregulated DEGs were mainly enriched in organic substance metabolic process $(\mathrm{P}=2.93 \mathrm{E}-06)$. In the E15.5 group, the upregulated DEGs were mainly enriched in cellular process $(\mathrm{P}=2.64 \mathrm{E}-19)$ and the downregulated DEGs were mainly enriched in cellular process $(\mathrm{P}=2.01 \mathrm{E}-19)$. Besides, we also performed the GO function enrichment analysis for the genes that were shared by E13.5 group and E15.5 group. The results showed that the upregulated DEGs were mainly related to cellular process $(\mathrm{P}=0.00650)$ and the downregulated DEGs were mainly related to organic substance metabolic process $(\mathrm{P}=0.00031)$ (Fig. 1).

KEGG and Reactome pathway analysis of DEGs. KEGG and Reactome enrichment pathway analysis were performed to explore the enriched pathways of the DEGs. The KEGG enrichment analysis results showed that the upregulated DEGs in E13.5 group were enriched in 64 pathways, such as Icam1, H2-M9, and 4930468A15Rik were enriched in cell adhesion molecules (CAMs) ( $\mathrm{P}=1.56 \mathrm{E}-03)$. The downregulated DEGs in E13.5 group were enriched in 84 pathways, such as Bmprla, Tgfbrl and Smurfl were enriched in TGF- $\beta$ signaling pathway $(\mathrm{P}=4.03 \mathrm{E}-03)$. The upregulated DEGs in E15.5 group were enriched in 129 pathways, such as Icaml, Cls and Fcgr $2 b$ were enriched in Staphylococcus aureus infection $(\mathrm{P}=1.80 \mathrm{E}-04)$. Meanwhile, the downregulated DEGs in E15.5 group were enriched in 156 pathways, such as Erollb, Dnajc3 and Dnajbl1 were enriched in protein processing in endoplasmic reticulum $(\mathrm{P}=1.27 \mathrm{E}-03)$. Moreover, the shared DEGs that were upregulated such as Cmah and Gmppa were enriched in nucleotide sugar metabolism $(\mathrm{P}=2.65 \mathrm{E}-03)$. And the shared DEGs that were downregulated such as Akt3, Pak3 and $\mathrm{Hgf}$ were enriched in renal cell carcinoma ( $\mathrm{P}=5.00 \mathrm{E}-04) ; A k t 3$, Pak3 and Pakl were enriched in T cell receptor signaling pathway ( $\mathrm{P}=2.60 \mathrm{E}-03)$ (Table $\mathrm{I})$. Reactome enrichment analysis results showed that the upregulated DEGs in E13.5 group were enriched in 154 pathways and the downregulated DEGs in E13.5 group were enriched in 192 pathways. The upregulated DEGs in E15.5 group were enriched in 27 pathways 

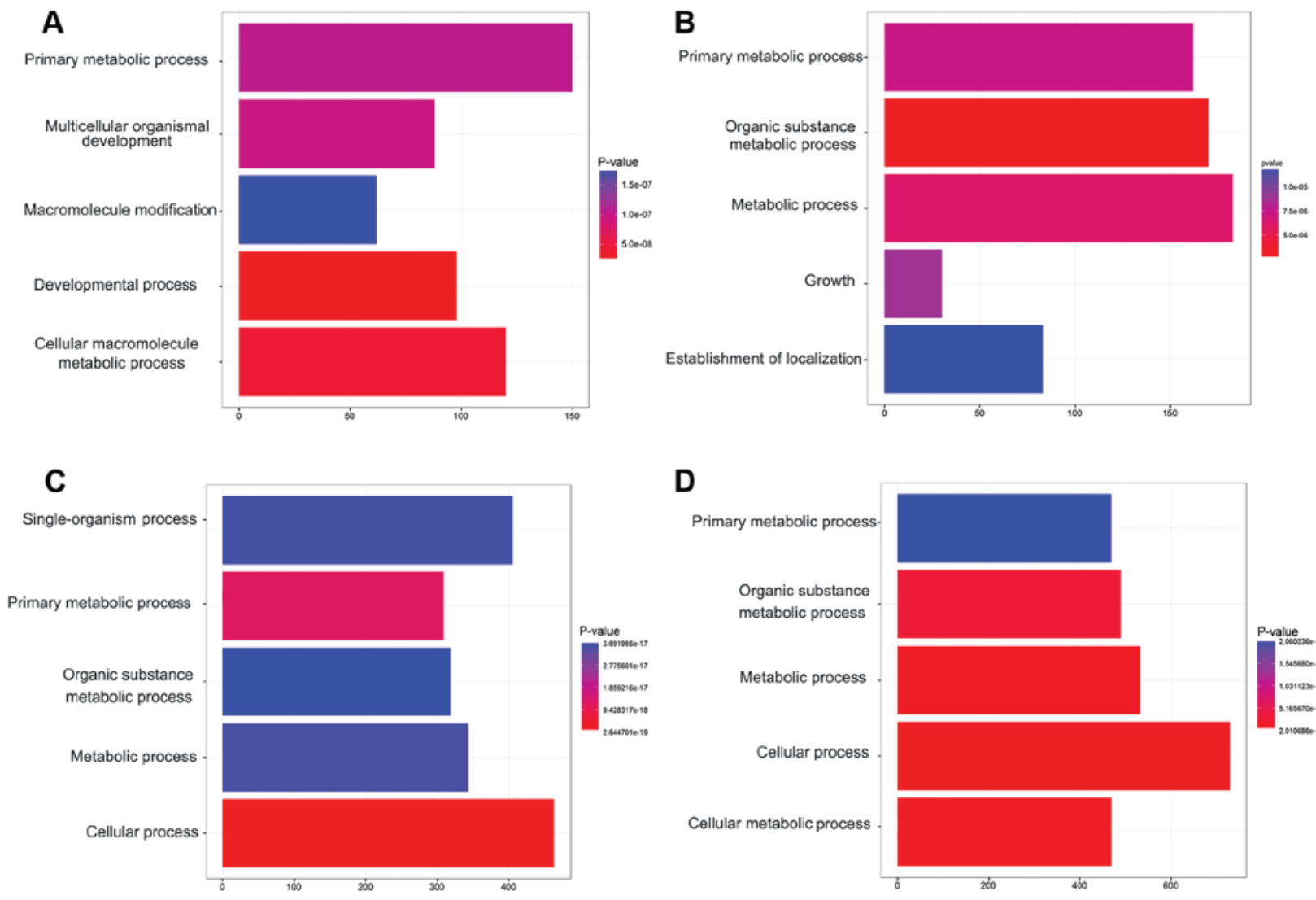

D
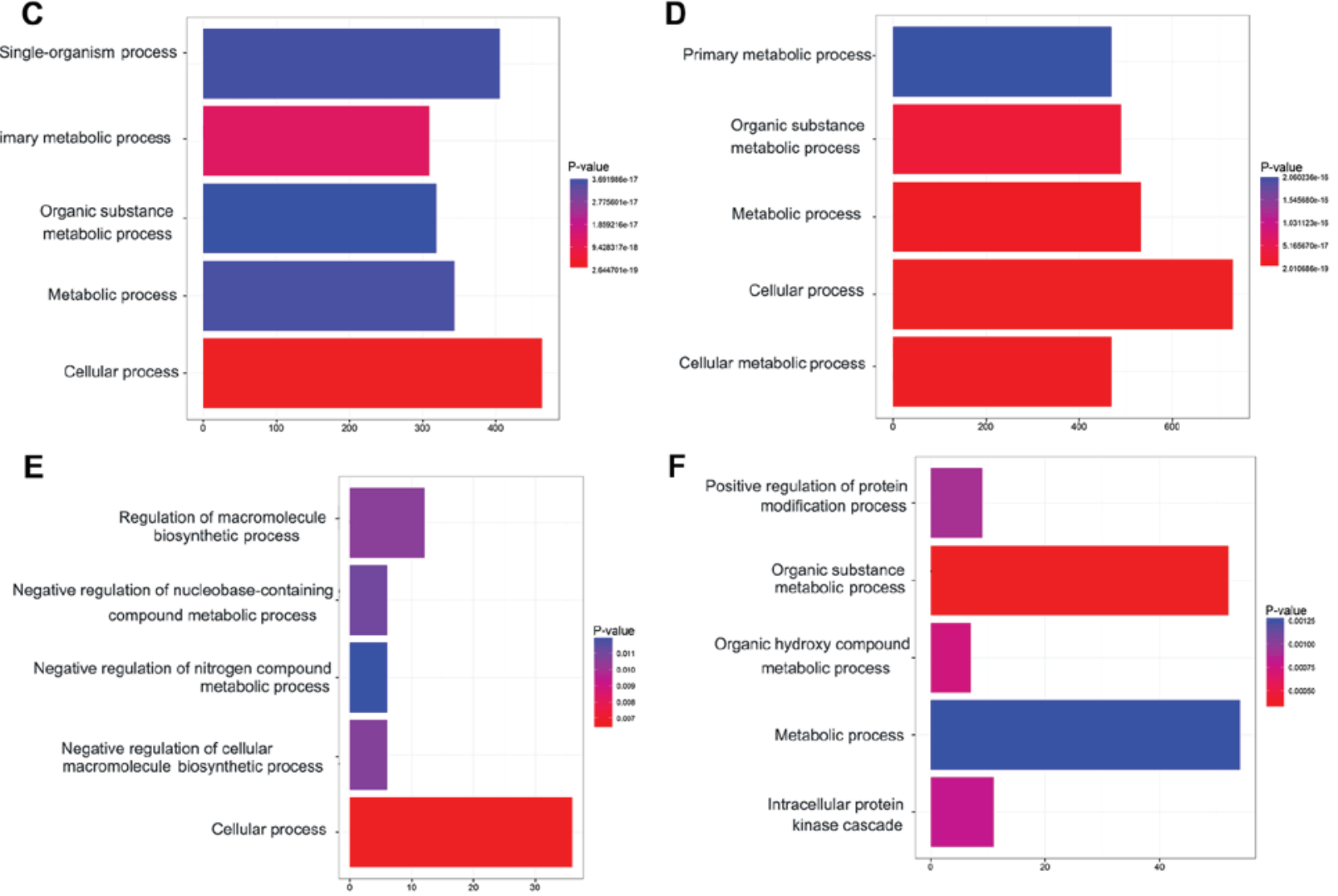

$\mathbf{F}$

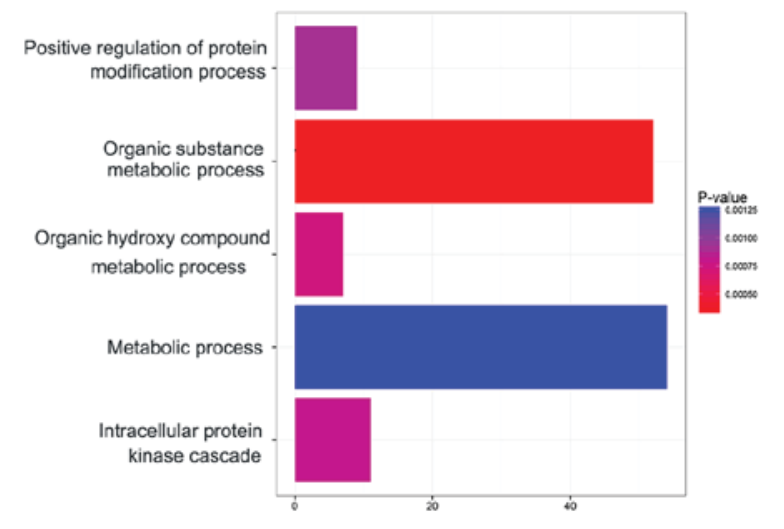

Figure 1. GO function analysis of DEGs in the developing hearts of embryos from diabetic mice. (A) GO function analysis of upregulated DEGs in E13.5 group; (B) GO function analysis of downregulated DEGs in E13.5 group; (C) GO function analysis of upregulated DEGs in E15.5 group; (D) GO function analysis of downregulated DEGs in E15.5 group; (E) GO function analysis of upregulated DEGs in E13.5 group and E15.5 group; and (F) GO function analysis of downregulated DEGs both in E13.5 group and E15.5 group. GO, gene ontology; DEGs, differentially expressed genes; E13.5, embryonic day 13.5; E15.5, embryonic day 15.5 .

and the downregulated DEGs in E15.5 group were enriched in 24 pathways. In addition, the shared DEGs that were upregulated such as Nr6al and Esrrg were enriched in nuclear receptor transcription pathway $(\mathrm{P}=5.09 \mathrm{E}-03)$ and the shared DEGs that were downregulated such as Pak3 and Pakl were enriched in activation of Rac ( $\mathrm{P}=1.85 \mathrm{E}-03)$ (Table II).

PPI network construction. STRING was used to construct the PPI networks for DEGs. The PPI networks for up- and downregulated DEGs in E13.5 group contained 114 and 112 nodes, respectively (Fig. 2); the PPI networks for up- and downregulated DEGs in E15.5 group contained 345 and 562 nodes, respectively (Fig. 3). The proteins such as Icam1,
Akp3, Stat1 and Brca1 had high connectivity degrees in the PPI networks of E13.5 (Table III) and E15.5 (Table IV) groups. Additionally, the PPI network for shared DEGs contained 17 nodes and 12 PPI pairs (Fig. 4). The shared DEGs with top three connectivity degrees of the PPI network were Icam1, Ncorl and Akt3 (Table V).

\section{Discussion}

Maternal diabetes is a relatively common disease that results in an increased incidence of congenital malformations such as neural tube defects and heart defects (9). CHD are the most common type of birth defects and a main cause of 
Table I. Enrichment analysis of top five KEGG pathways for DEGs.

\begin{tabular}{|c|c|c|c|c|c|}
\hline DEGs & Term & Description & P-value & Gene ID & Count \\
\hline \multirow[t]{5}{*}{$\begin{array}{l}\text { Upregulated } \\
\text { DEGs in E13.5 }\end{array}$} & $\mathrm{mmu} 04514$ & $\begin{array}{l}\text { Cell adhesion } \\
\text { molecules (CAMs) }\end{array}$ & $1.56 \times 10^{-03}$ & $\begin{array}{l}\text { Icam1, H2-M9, 4930468A15Rik, H2-D1, } \\
\text { Itga4, H2-Ob, Cntnap2, Nrxn2 }\end{array}$ & 8 \\
\hline & $\mathrm{mmu} 04672$ & $\begin{array}{l}\text { Intestinal immune network } \\
\text { for IgA production }\end{array}$ & $3.77 \times 10^{-03}$ & Gm13306, Itga4, Ill15, H2-Ob & 4 \\
\hline & mmu05330 & Allograft rejection & $8.24 \times 10^{-03}$ & $H 2-M 9, H 2-D 1, H 2-O b, I l 12 b$ & 4 \\
\hline & mmu04940 & Type I diabetes mellitus & $1.24 \times 10^{-02}$ & $H 2-M 9, H 2-D 1, H 2-O b, I l 12 b$ & 4 \\
\hline & mmu04146 & Peroxisome & $2.74 \times 10^{-02}$ & Agps, Nudt19, Slc25a17, Acsbg2 & 4 \\
\hline \multirow[t]{5}{*}{$\begin{array}{l}\text { Downregulated } \\
\text { DEGs in E13.5 }\end{array}$} & mmu04350 & TGF- $\beta$ signaling pathway & $4.03 \times 10^{-03}$ & $\begin{array}{l}\text { Bmprla, Tgfbr1, Smurf1, Ltbp1, } \\
\text { Lefty2, Thbs } 2\end{array}$ & 6 \\
\hline & mmu05211 & Renal cell carcinoma & $9.14 \times 10^{-03}$ & Akt3, Pak3, Hgf, Pak1, Pik3ca & 5 \\
\hline & mmu04080 & $\begin{array}{l}\text { Neuroactive ligand-receptor } \\
\text { interaction }\end{array}$ & $1.05 \times 10^{-02}$ & $\begin{array}{l}\text { Prlr, Gabra1, Tshr, Gabra2, } \\
\text { Gabrb2, Crhr } 2 \ldots\end{array}$ & 11 \\
\hline & mmu05223 & Non-small cell lung cancer & $1.66 \times 10^{-02}$ & Akt3, Pik3ca, Stk4, Rarb & 4 \\
\hline & mmu04210 & Apoptosis & $1.87 \times 10^{-02}$ & Irak3, Il3, Akt3, Endod1, Pik3ca & 5 \\
\hline \multirow[t]{5}{*}{$\begin{array}{l}\text { Upregulated } \\
\text { DEGs in E15.5 }\end{array}$} & mmu05150 & $\begin{array}{l}\text { Staphylococcus aureus } \\
\text { infection }\end{array}$ & $1.80 \times 10^{-04}$ & $\begin{array}{l}\text { Icam } 1, C 1 s, F c g r 2 b, H 2-A b 1 \\
C 2, F p r 2, C f h, H 2-A a\end{array}$ & 8 \\
\hline & $\mathrm{mmu} 04514$ & $\begin{array}{l}\text { Cell adhesion molecules } \\
\text { (CAMs) }\end{array}$ & $1.18 \times 10^{-03}$ & Icam1, Vcan, H2-K1, Cd80, Ncam1 ... & 13 \\
\hline & mmu04145 & Phagosome & $1.61 \times 10^{-03}$ & $\begin{array}{l}\text { Lamp2, H2-K1, Tap2, Tuba4a, } \\
\text { Thbs } 2, F \operatorname{cgr} 2 b \ldots\end{array}$ & 14 \\
\hline & mmu05219 & Bladder cancer & $2.20 \times 10^{-03}$ & $R b 1, M m p 9, M y c, E 2 f 3, E g f r, E 2 f 2$ & 6 \\
\hline & mmu05220 & Chronic myeloid leukemia & $2.27 \times 10^{-03}$ & $\begin{array}{l}\text { Bcl2l1, Rb1, Myc, Cblb, E2f3, } \\
\text { Sos2,E2f2, Abl1 }\end{array}$ & 8 \\
\hline \multirow[t]{5}{*}{$\begin{array}{l}\text { Downregulated } \\
\text { DEGs in E15.5 }\end{array}$} & mmu04141 & $\begin{array}{l}\text { Protein processing in } \\
\text { endoplasmic reticulum }\end{array}$ & $1.27 \times 10^{-03}$ & $\begin{array}{l}\text { Erollb, Dnajc3, Dnajb11, Sec62, } \\
\text { Derl1, Mapk8... }\end{array}$ & 18 \\
\hline & mmu01100 & Metabolic pathways & $2.25 \times 10^{-03}$ & $\begin{array}{l}\text { Phgdh, B3gnt5, Sucla2, Alad, } \\
\text { B4galt6, Mmab... }\end{array}$ & 77 \\
\hline & mmu04977 & $\begin{array}{l}\text { Vitamin digestion } \\
\text { and absorption }\end{array}$ & $4.84 \times 10^{-03}$ & Tcn2, Rbp2, Plb1, Lrat, Slc5a6 & 5 \\
\hline & mmu04010 & MAPK signaling pathway & $9.22 \times 10^{-03}$ & $\begin{array}{l}\text { Cacnala, Ptpn5, Mapk8, Cacna2d1, } \\
\text { Akt3, Gna12... }\end{array}$ & 22 \\
\hline & $\mathrm{mmu} 00512$ & $\begin{array}{l}\text { Mucin type O-Glycan } \\
\text { biosynthesis }\end{array}$ & $9.57 \times 10^{-03}$ & Gcnt3, C1galt1, Galnt2, Galnt7, Galnt15 & 5 \\
\hline \multirow[t]{2}{*}{$\begin{array}{l}\text { Upregulated } \\
\text { genes in shared } \\
\text { DEGs }\end{array}$} & $\mathrm{mmu} 00520$ & $\begin{array}{l}\text { Amino sugar and } \\
\text { nucleotide sugar } \\
\text { metabolism }\end{array}$ & $2.65 \times 10^{-03}$ & Cmah, Gmppa & 2 \\
\hline & mmu01100 & Metabolic pathways & $6.08 \times 10^{-01}$ & Lias, Gmppa & 2 \\
\hline \multirow{10}{*}{$\begin{array}{l}\text { Downregulated } \\
\text { genes in shared } \\
\text { DEGs }\end{array}$} & mmu05211 & Renal cell carcinoma & $5.00 \times 10^{-04}$ & Akt3, Pak3, Hgf, Pakl, & 4 \\
\hline & mmu04660 & $\begin{array}{l}\mathrm{T} \text { cell receptor signaling } \\
\text { pathway }\end{array}$ & $2.60 \times 10^{-03}$ & Akt3, Pak3, Pak1, Grap2 & 4 \\
\hline & mmu04730 & Long-term depression & $6.09 \times 10^{-03}$ & Cacnala, Gna12, Ppplr17 & 3 \\
\hline & mmu04210 & Apoptosis & $9.95 \times 10^{-03}$ & Akt3, Il3, Endodl & 3 \\
\hline & mmu04012 & ErbB signaling pathway & $1.03 \times 10^{-02}$ & Akt3, Pak3, Pakl & 3 \\
\hline & mmu04510 & Focal adhesion & $1.96 \times 10^{-02}$ & Akt3, Pak3, Hgf, Pakl, & 4 \\
\hline & $\mathrm{mmu04010}$ & MAPK signaling pathway & $5.02 \times 10^{-02}$ & Akt3, Cacnala, Gnal2, Pakl & 4 \\
\hline & mmu04080 & $\begin{array}{l}\text { Neuroactive ligand-receptor } \\
\text { interaction }\end{array}$ & $5.48 \times 10^{-02}$ & Prlr, Tshr, Gabrb2, Crhr2 & 4 \\
\hline & mmu04630 & Jak-STAT signaling pathway & $4.49 \times 10^{-02}$ & Akt3, Prlr, Il3 & 3 \\
\hline & mmu00140 & Steroid hormone biosynthesis & $3.31 \times 10^{-02}$ & $H s d 17 b 7, H s d 11 b 2$ & 2 \\
\hline
\end{tabular}


Table II. Enrichment analysis of top five Reactome pathways for DEGs.

\begin{tabular}{|c|c|c|c|c|}
\hline DEGs & Term & Description & P-value & Gene ID \\
\hline \multirow{5}{*}{$\begin{array}{l}\text { Upregulated } \\
\text { DEGs in E13.5 }\end{array}$} & 4809882 & Neuronal system & $2.10 \times 10^{-03}$ & Gjd2, Braf, Kcnb1, Chrna3, Gabrg2, Gls... \\
\hline & 4810204 & $\begin{array}{l}\text { Neurotransmitter receptor } \\
\text { binding and downstream } \\
\text { transmission in the } \\
\text { post-synaptic cell }\end{array}$ & $2.14 \times 10^{-03}$ & $\begin{array}{l}\text { Braf, Chrna3, Gabrg2, Arhgef9, Ap2a2, } \\
\text { Epb4.1l1, Rps6ka2 }\end{array}$ \\
\hline & 4810917 & Interferon $\gamma$ signaling & $2.64 \times 10^{-03}$ & Icam1, Oas1h, Irf7, Sp100, H2-D1 \\
\hline & 4809881 & $\begin{array}{l}\text { Transmission across } \\
\text { chemical synapses }\end{array}$ & $3.10 \times 10^{-03}$ & $\begin{array}{l}\text { Braf, Chrna3, Gabrg2, Gls, Arhgef9, } \\
\text { Ap2a2, Epb4.1l1, Rps6ka2 }\end{array}$ \\
\hline & 4810848 & $\begin{array}{l}\text { Regulation of cytoskeletal } \\
\text { remodeling and cell spreading } \\
\text { by IPP complex components }\end{array}$ & $5.26 \times 10^{-03}$ & Parvb, Pxn \\
\hline \multirow[t]{5}{*}{$\begin{array}{l}\text { Downregulated } \\
\text { DEGs in E13.5 }\end{array}$} & 4810408 & GABA receptor activation & $3.56 \times 10^{-04}$ & $\begin{array}{l}\text { Gabra1, Gabra2, Gabrb2, Gngt2, } \\
\text { Kcnj3, Kcnj9 }\end{array}$ \\
\hline & 4809881 & $\begin{array}{l}\text { Transmission across } \\
\text { chemical synapses }\end{array}$ & $8.56 \times 10^{-04}$ & $\begin{array}{l}\text { Cacnala, Gabral, Gabra2, Gabrb2, } \\
\text { Camk2b, Gngt } 2 . .\end{array}$ \\
\hline & 4809882 & Neuronal system & $9.71 \times 10^{-04}$ & $\begin{array}{l}\text { Cacnala, Gabra1, Gabra2, Gabrb2, } \\
\text { Camk2b, Kcnq5... }\end{array}$ \\
\hline & 4810957 & GABA A receptor activation & $1.01 \times 10^{-03}$ & Gabra1, Gabra2, Gabrb2 \\
\hline & 4810204 & $\begin{array}{l}\text { Neurotransmitter receptor } \\
\text { binding and downstream } \\
\text { transmission in the } \\
\text { post-synaptic cell }\end{array}$ & $1.68 \times 10^{-03}$ & $\begin{array}{l}\text { Gabra1, Gabra2, Gabrb2, Camk2b, } \\
\text { Gngt2, Kcnj3, Kcnj9, Chrnb3 }\end{array}$ \\
\hline \multirow[t]{5}{*}{$\begin{array}{l}\text { Upregulated } \\
\text { DEGs in E15.5 }\end{array}$} & 4810016 & Immune system & $1.13 \times 10^{-07}$ & $\begin{array}{l}\text { Ifitm3, Ifitm6, Lgals3, H2-K1, } \\
\text { Tap2, Bcl2l1 ... }\end{array}$ \\
\hline & 4810918 & Interferon signaling & $5.69 \times 10^{-05}$ & $\begin{array}{l}\text { Icam1, Stat1, Irfl, Oas } 1 h, \\
\text { Ncam1, Ifnar } 2 . .\end{array}$ \\
\hline & 4810008 & $\begin{array}{l}\text { EGFR interacts with } \\
\text { phospholipase } C-\gamma\end{array}$ & $5.88 \times 10^{-05}$ & $\begin{array}{l}\text { Adcy7, Plcg1, Adrbk1, Egfr, } \\
\text { Creb1, Camk4, Adcy9 }\end{array}$ \\
\hline & 4810917 & Interferon $\gamma$ signaling & $6.00 \times 10^{-05}$ & $\begin{array}{l}\text { Icam1, Stat1, H2-K1, Irf1, } \\
\text { Oas1h, Ncam1, Socs3... }\end{array}$ \\
\hline & 4810011 & $\begin{array}{l}\text { PLCG1 events in } \\
\text { ERBB2 signaling }\end{array}$ & $7.26 \times 10^{-05}$ & $\begin{array}{l}\text { Adcy7, Plcg1, Adrbk1, Egfr, } \\
\text { Creb1, Camk4, Adcy9 }\end{array}$ \\
\hline \multirow[t]{5}{*}{$\begin{array}{l}\text { Downregulated } \\
\text { DEGs in E15.5 }\end{array}$} & 4809896 & $\begin{array}{l}\text { Metabolism of water-soluble } \\
\text { vitamins and cofactors }\end{array}$ & $2.69 \times 10^{-03}$ & $\begin{array}{l}\text { Amn, Mmaa, Mmab, Tcn2, Slc23a2, } \\
\text { Mthfr, Rfk, Slc5a6, Mocs1, Ppcs }\end{array}$ \\
\hline & 4809897 & $\begin{array}{l}\text { Metabolism of vitamins } \\
\text { and cofactors }\end{array}$ & $2.69 \times 10^{-03}$ & $\begin{array}{l}\text { Amn, Mmaa, Mmab, Tcn2, Slc23a2, } \\
\text { Mthfr, Rfk, Slc5a6, Mocs1, Ppcs }\end{array}$ \\
\hline & 4809898 & $\begin{array}{l}\text { Defective TCN2 causes } \\
\text { hereditary megaloblastic } \\
\text { anemia }\end{array}$ & $2.69 \times 10^{-03}$ & $\begin{array}{l}\text { Amn, Mmaa, Mmab, Tcn2, Slc23a2, } \\
\text { Mthfr, Rfk, Slc5a6, Mocs 1, Ppcs }\end{array}$ \\
\hline & 4809899 & $\begin{array}{l}\text { Defects in cobalamin (B12) } \\
\text { metabolism }\end{array}$ & $2.69 \times 10^{-03}$ & $\begin{array}{l}\text { Amn, Mmaa, Mmab, Tcn2, Slc23a2, } \\
\text { Mthfr, Rfk, Slc5a6, Mocs 1, Ppcs }\end{array}$ \\
\hline & 4809900 & $\begin{array}{l}\text { Defects in vitamin and } \\
\text { cofactor metabolism }\end{array}$ & $2.69 \times 10^{-03}$ & $\begin{array}{l}\text { Amn, Mmaa, Mmab, Tcn2, Slc23a2, } \\
\text { Mthfr, Rfk, Slc5a6, Mocs1, Ppcs }\end{array}$ \\
\hline $\begin{array}{l}\text { Upregulated } \\
\text { genes in shared }\end{array}$ & 4810720 & $\begin{array}{l}\text { Nuclear receptor } \\
\text { transcription pathway }\end{array}$ & $5.09 \times 10^{-03}$ & Nr6al, Esrrg \\
\hline \multirow[t]{4}{*}{ DEGs } & 4810917 & Interferon $\gamma$ signaling & $9.26 \times 10^{-03}$ & Icam1, Oas1h \\
\hline & 4810918 & Interferon signaling & $2.35 \times 10^{-02}$ & Icam1, Oas1h \\
\hline & 4810429 & $\begin{array}{l}\text { Generic transcription } \\
\text { pathway }\end{array}$ & $3.07 \times 10^{-02}$ & Nr6al, Esrrg \\
\hline & 4810016 & Immune system & $6.30 \times 10^{-02}$ & Icam1, Oas1h, C6, Fbxw11, Kif2a \\
\hline
\end{tabular}


Table II. Continued.

\begin{tabular}{llllr}
\hline DEGs & Term & \multicolumn{1}{c}{ Description } & P-value & Gene ID \\
\hline Downregulated & 4810839 & Activation of Rac & $1.85 \times 10^{-03}$ & Pak3, Pakl \\
genes in shared & 4810573 & CD28 co-stimulation & $5.02 \times 10^{-03}$ & Pakl, Grap2 \\
DEGs & 4810613 & Generation of second & $5.02 \times 10^{-03}$ & Pakl, Grap2 \\
& & messenger molecules & & 2 \\
& 4810625 & Signaling by Robo receptor & $9.02 \times 10^{-03}$ & Pak3, Pakl \\
& 4810145 & FCERI mediated & $1.55 \times 10^{-02}$ & Pakl, Grap2
\end{tabular}

E13.5, embryonic day 13.5; E15.5, embryonic day 15.5; DEGs, differentially expressed genes.

Table III. DEGs with the top 10\% connectivity degree in the PPI network in E13.5 group.

\begin{tabular}{|c|c|c|c|c|c|c|c|c|}
\hline DEGs & ID & Degree & ID & Degree & ID & Degree & ID & Degree \\
\hline \multirow[t]{4}{*}{ Upregulated DEGs } & Icam1 & 9 & H2-M9 & 5 & Ube2t & 4 & Calb1 & 3 \\
\hline & H2-L & 8 & Gabrg2 & 5 & Sgip1 & 4 & Itga4 & 3 \\
\hline & $\operatorname{Irf7}$ & 7 & Pxn & 5 & Ap2a2 & 3 & & \\
\hline & Oas1h & 5 & $\mathrm{Il} 12 \mathrm{~b}$ & 4 & Rnf2 & 3 & & \\
\hline \multirow[t]{3}{*}{ Downregulated DEGs } & Akp3 & 13 & Ncor1 & 7 & Pik3ca & 6 & Akt3 & 5 \\
\hline & $\mathrm{Nr} 3 \mathrm{c} 1$ & 10 & Stat2 & 6 & Bmprla & 6 & Gna15 & 5 \\
\hline & Ar & 9 & $\mathrm{Il} 3$ & 6 & Gngt2 & 5 & Mut & 4 \\
\hline
\end{tabular}

DEGs, differentially expressed genes; PPI, protein-protein interaction; E13.5, embryonic day 13.5.

Table IV. DEGs with the top $10 \%$ connectivity degree in the PPI network in E15.5 group.

\begin{tabular}{|c|c|c|c|c|c|c|c|c|c|c|}
\hline DEGs & ID & Degree & ID & Degree & ID & Degree & ID & Degree & ID & Degree \\
\hline \multirow[t]{7}{*}{ Upregulated DEGs } & Stat1 & 38 & Mmp9 & 19 & Ifih1 & 16 & Ezr & 13 & Lgals3bp & 12 \\
\hline & Egfr & 37 & Irf1 & 18 & Cxcl1 & 15 & Mbp & 13 & Oasl2 & 12 \\
\hline & Icam1 & 27 & Ccr2 & 18 & Ifi35 & 14 & Hnrnpa1 & 13 & Rbm25 & 12 \\
\hline & Myc & 27 & Fpr2 & 18 & Actb & 14 & Rmcs2 & 13 & Ccr9 & 12 \\
\hline & Pten & 25 & Ncam1 & 17 & Ccl6 & 14 & Cpsf6 & 13 & $\mathrm{H} 2-\mathrm{Aa}$ & 12 \\
\hline & Stat2 & 21 & Plcg1 & 17 & Cblb & 14 & Adrbk1 & 12 & Abl1 & 12 \\
\hline & Yes1 & 20 & Creb1 & 16 & $\mathrm{H} 2-\mathrm{K} 1$ & 14 & Rsad2 & 12 & & \\
\hline \multirow[t]{11}{*}{ Downregulated DEGs } & Brca1 & 33 & Dlgap5 & 20 & Tmem48 & 17 & Rpn1 & 12 & Haol & 10 \\
\hline & Chek1 & 30 & Creb1 & 20 & Hspa5 & 16 & Kcnj11 & 12 & Ugt2b5 & 10 \\
\hline & Bub1 & 29 & Aspm & 20 & Lsm4 & 14 & Med1 & 11 & Abcb11 & 10 \\
\hline & Rrm2 & 26 & Zwint & 20 & Akt3 & 14 & Calr & 11 & Gnao1 & 10 \\
\hline & Sgol2 & 25 & Cenpm & 19 & Sec61a1 & 13 & Kalrn & 11 & Gabrg2 & 10 \\
\hline & Cep55 & 25 & Spc24 & 19 & Lpar3 & 13 & Slc10a1 & 11 & $\mathrm{Cd} 40$ & 10 \\
\hline & Plk4 & 24 & Eme1 & 19 & Hjurp & 13 & Rgn & 10 & Chrm4 & 10 \\
\hline & Tpx2 & 24 & Foxm1 & 19 & Nasp & 13 & Cfi & 10 & Ppp2r1a & 9 \\
\hline & Kif 23 & 23 & Xpo1 & 18 & Igf1r & 13 & Ddost & 10 & Nfatc2 & 9 \\
\hline & Ska1 & 23 & Troap & 18 & Gucy $1 b 3$ & 13 & Prkx & 10 & Hpd & 9 \\
\hline & Prc1 & 23 & Oip5 & 17 & Ncor1 & 12 & Rae1 & 10 & Fanci & 9 \\
\hline
\end{tabular}


A

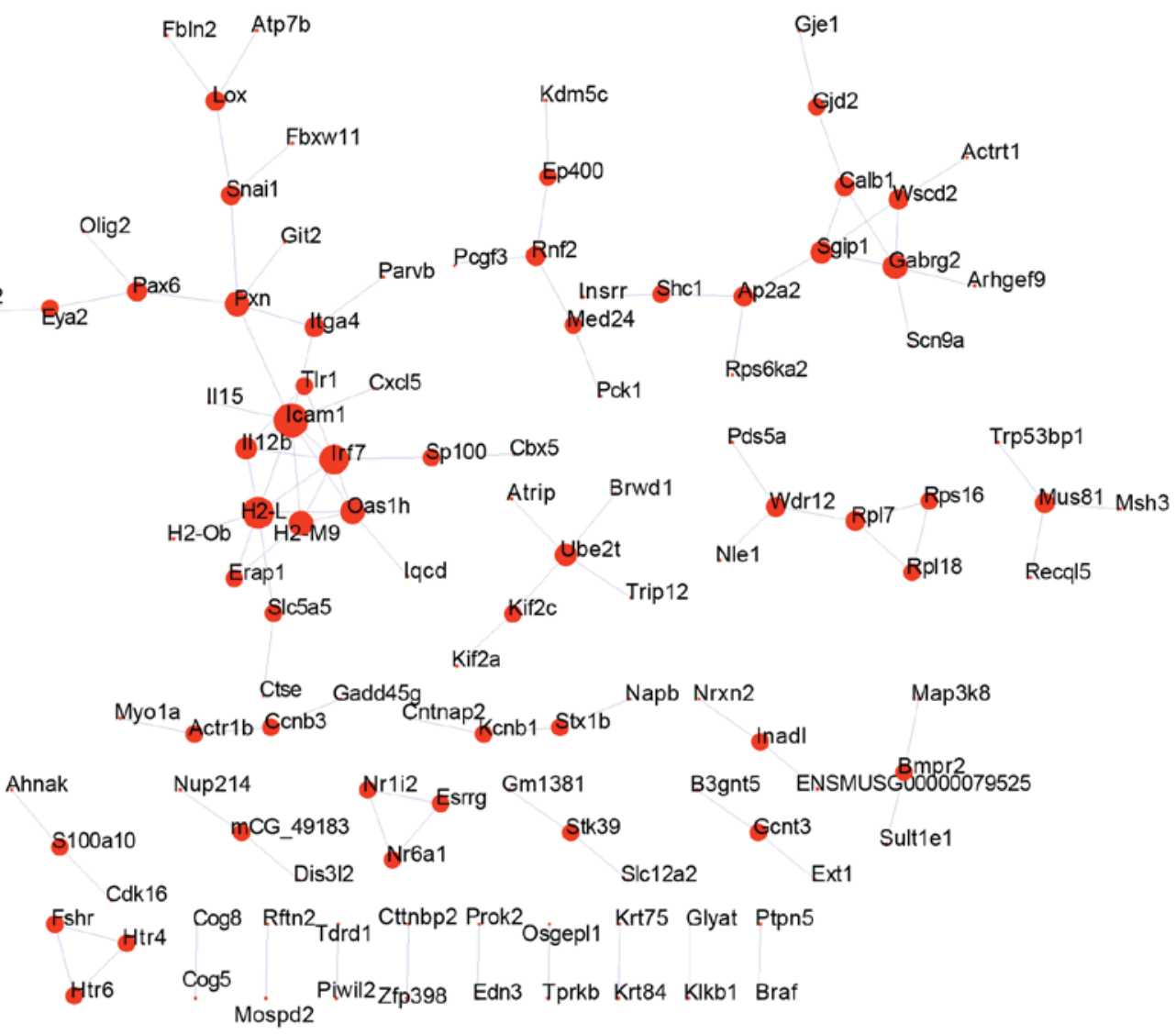

B

Slc27a2

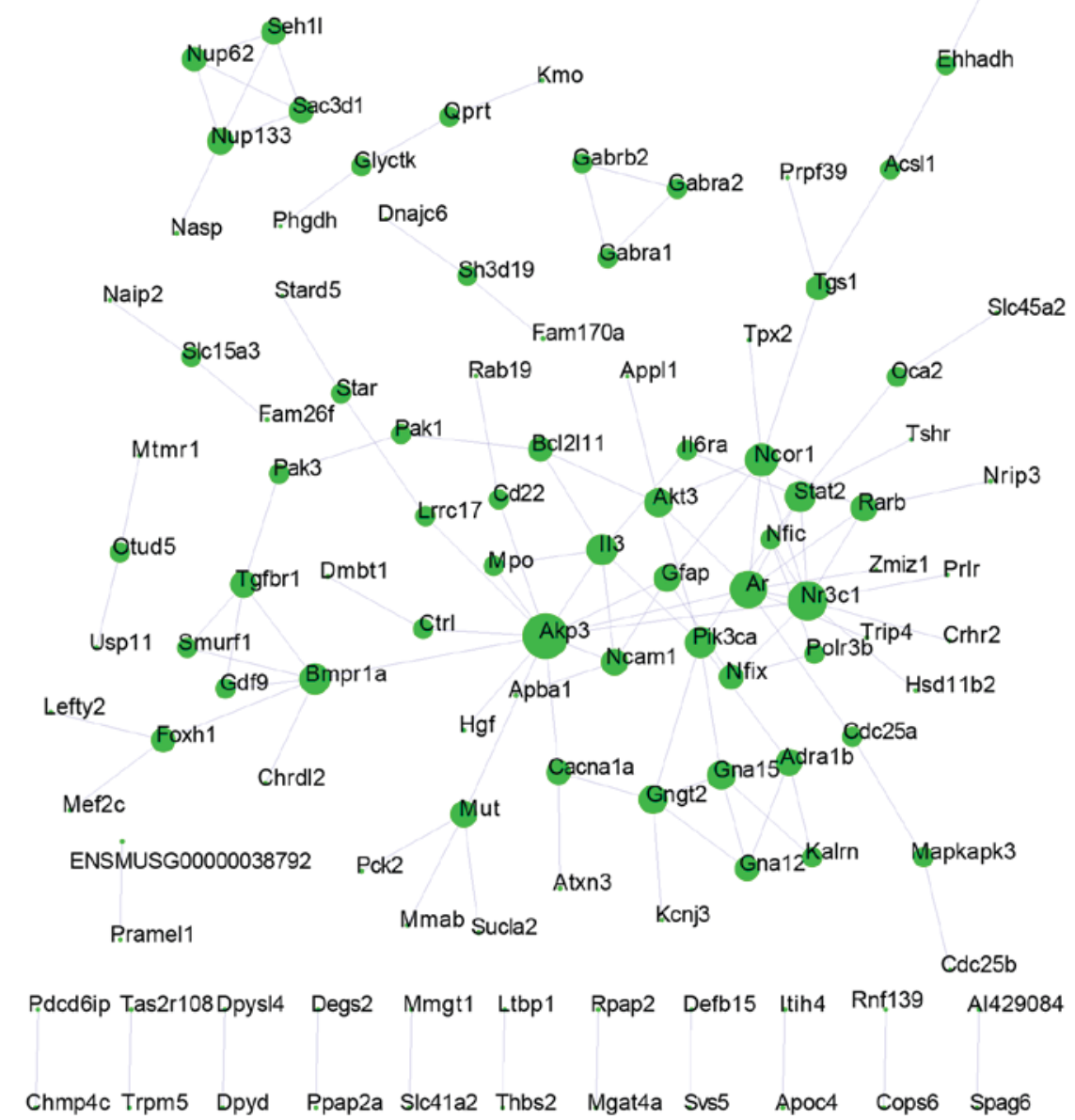

Figure 2. PPI networks for upregulated (A) and downregulated (B) DEGs in E13.5 hearts of embryos from diabetic mice. The size of node indicates the degree of DEG. DEGs, differentially expressed genes; PPI, protein-protein interaction; E13.5, embryonic day 13.5. 
A

A
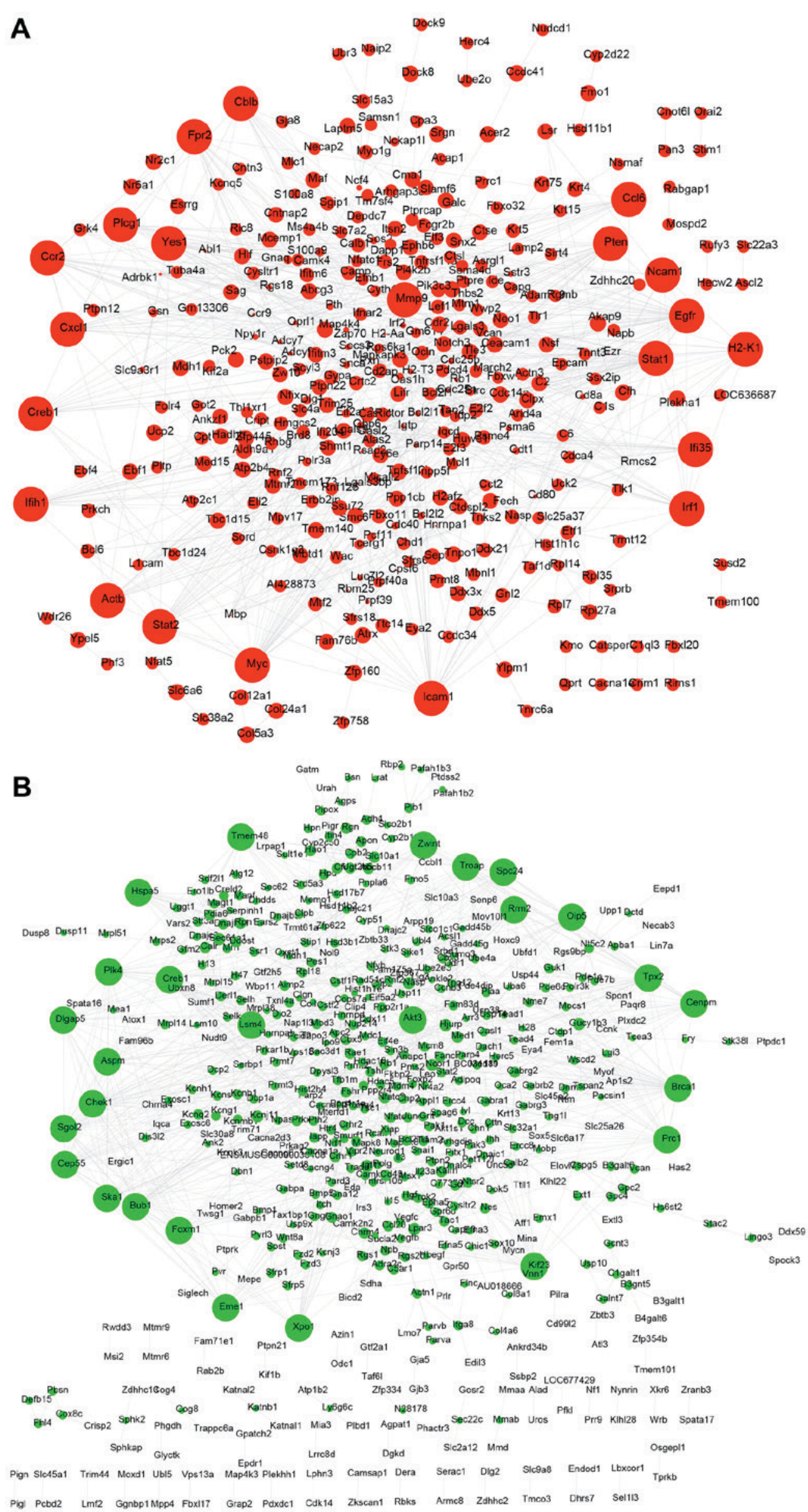

Figure 3. PPI networks for upregulated (A) and downregulated (B) DEGs in E15.5 hearts of embryos from diabetic mice. The size of node indicates the degree of DEG. DEGs, differentially expressed genes; PPI, protein-protein interaction; E15.5, embryonic day 15.5. 
Table V. Connectivity degrees in the PPI network for shared DEGs in E13.5 and E15.5 groups.

\begin{tabular}{lclclccc}
\hline ID & Degree & ID & Degree & ID & Degree & ID & Degree \\
\hline Icam1 & 3 & Oca2 & 1 & Hgf & 1 & Tpx2 & 1 \\
Ncor1 & 3 & Slc45a2 & 1 & Esrrg & 1 & Il3 & 1 \\
Akt3 & 2 & App11 & 1 & Pak3 & 1 & & \\
Oas1h & 2 & Sgip1 & 1 & Pak1 & 1 & & \\
Nr6a1 & 2 & Calb1 & 1 & Iqcd & 1 & & \\
\hline
\end{tabular}

DEGs, differentially expressed genes; PPI, protein-protein interaction; E13.5, embryonic day 13.5; E15.5, embryonic day 15.5.

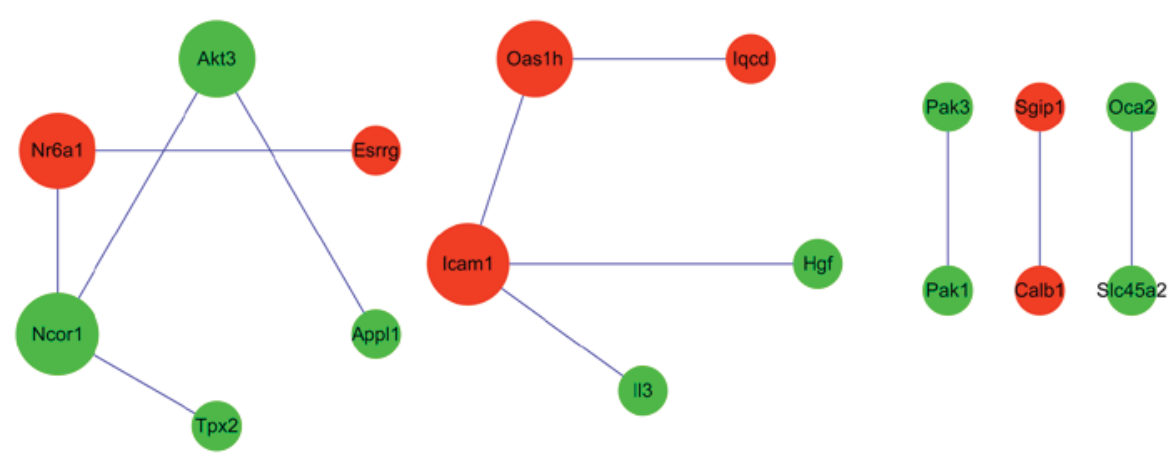

Figure 4. PPI networks for DEGs both in E13.5 and E15.5 hearts of embryos from diabetic mice. The red and green nodes respectively indicate up- and downregulated DEGs. The size of node indicates the degree of DEG. DEGs, differentially expressed genes; PPI, protein-protein interaction; E13.5, embryonic day 13.5; E15.5, embryonic day 15.5 .

birth defects-related mortality and morbidity $(24,25)$. It has been reported that $\mathrm{CHD}$ are closely associated with maternal diabetes (26). Previous study had screened and analyzed several DEGs in embryonic heart tissues from diabetic mother (4). However, the molecular mechanism between CHD and diabetes remains largely unknown. In this study, we have screened 869 and 1,295 in E13.5 and E15.5 groups, respectively and 182 DEGs were shared by two groups. Moreover, the DEGs such as Icaml and H2-M9 were significantly enriched in cell adhesion molecules (CAMs); DEGs such as Bmprla, Tgfbrl and Smurfl were enriched in TGF- $\beta$ signaling pathway; DEGs such as Icam1, Cls and Fcgr2b were enriched in Staphylococcus aureus infection. Finally, several key shared DEGs that were the genes with the top three node degrees in the network were analyzed, including Icaml, Ncorl and Akt3.

Our results showed that Icaml had the highest connectivity degree not only in the PPI network of the upregulated DEGs in E13.5 group but also in the PPI network of the shared DEGs. ICAM1, also called CD54, is a cell surface glycoprotein which is typically expressed on endothelial cells and cells of the immune system (27) and the protein is considered to participate in atherogenesis by promoting monocyte accumulation in the arterial intima (28). An earlier study has found that the expression of ICAMI on endothelial cells and circulating monocytes may be critical for the adhesion of the cells on the vascular endothelium (29). And the expression of ICAMI has been also detected on cardiac myocytes both in adult humans with unexplained cardiac dysfunction (30) and animals with myocarditis $(31,32)$. According to the pathway enrichment results in our study, we found that Icaml were enriched in Staphylococcus aureus infection, CAMs and interferon $\gamma$ signaling. Inflammation is showed to participate in the pathogenesis of type 2 diabetes (33). Upregulation of adhesion molecules such as ICAMI is pivotal in the development of inflammatory responses (34). Besides, autoimmune-associated congenital heart block (CHB) may result from pathogenic cross-talk between inflammatory and profibrosing pathways (35). Therefore, Icaml might be involved in the development of heart in mouse embryos.

Ncorl also had a high node degree in the PPI network for the shared DEGs. It is well known that Ncorl is a transcriptional coregulator that controls the activity of many transcription factors (such as $M E F 2, E R R s$ ) and has wide-ranging effects on gene expression patterns (36). MEF2 family has been associated with regulation of myocardially-expressed genes within the heart, such as cardiac $\alpha$-actin (37). MEF2 is critical for normal heart development and mitochondrial integrity (38). There is a strict relationship between oxygen consumption and cardiac work (39). Oxidative stress is thought to play a particularly critical role in the development of cardiovascular pathology (40). SIRT1 that is part of Ncorl/SMRT complex (36) could retard aging and confer oxidative stress resistance to the heart in vivo $(41,42)$. SMRT (Ncor2), the homolog of Ncorl, is also considered to take part in heart formation (43). Thus, Ncorl, as a transcriptional coregulator, might regulate several genes that are related to the heart development through several different signaling pathways.

Akt3 was interacted with Ncorl in the PPI network for the shared DEGs. Study has showed that Akt3 is a member of the 
Akt subfamily that comprises three closely related isoforms Akt1, Akt2 and Akt3. Akt regulates several cellular process including metabolism, cell growth, proliferation, survival and angiogenesis (44). Dysregulation of $A k t$ leads to many diseases such as cancer, diabetes, cardiovascular and neurological diseases (44). Akt has an important role in the functional behavior in the cardiovasculature such as cardiomyocytes, thrombocytes and endothelial cells (44). Aktl is demonstrated to be essential for heart development and function (45). In addition, $A k t 3$ was found to be enriched in most of the KEGG pathways in shared DEGs, especially renal cell carcinoma. Renal insufficiency in patients with acquired heart failure and ischemic heart disease is related to higher mortality and morbidity (46). Therefore, Akt3 might to be related to the heart development during embryogenesis.

Additionally, there are some limitations in the present study. For example, much more samples should be used to clarify the finding; the expression level of Icaml, Ncorl, and Akt3 should be verified by RT-PCR in the maternal diabetes associated with CHD. However, the present study may provide a scientific guidance for future study to clarify the relationship between CHD and diabetes. It is helpful to explain the molecular mechanism of the CHD development in offspring of diabetic pregnancies. These DEGs may be the therapeutic target in the offspring of diabetic pregnancies with CHD.

In conclusion, we have identified many DEGs in embryonic heart tissue samples at E13.5 and E15.5 from diabetic mice using bioinformatics analysis. And we found that the shared DEGs such as Icaml, Ncorl, and Akt3 had high connectivity degrees in the network. Our study implied that maternal diabetes could affect Icaml, Ncorl, and Akt3 which are critical in the heart development during embryogenesis and might result in CHD. However, more research is needed to confirm these results and further explore the complex molecular mechanism.

\section{References}

1. Association AD: Diagnosis and classification of diabetes mellitus. Diabetes Care 33 (Supplement 1): S62-S69, 2010.

2. Rother KI: Diabetes treatment-bridging the divide. N Engl J Med 356: 1499-1501, 2007.

3. Kim C, Newton KM and Knopp RH: Gestational diabetes and the incidence of type 2 diabetes: A systematic review. Diabetes Care 25: 1862-1868, 2002.

4. Vijaya M, Manikandan J, Parakalan R, Dheen ST, Kumar SD and Tay SS: Differential gene expression profiles during embryonic heart development in diabetic mice pregnancy. Gene 516: 218-227, 2013.

5. Herrera E and Ortega-Senovilla H: Disturbances in lipid metabolism in diabetic pregnancy-are these the cause of the problem? Best Pract Res Clin Endocrinol Metab 24: 515-525, 2010.

6. Fahed AC, Gelb BD, Seidman JG and Seidman CE: Genetics of congenital heart disease: The glass half empty. Circ Res 112: 707-720, 2013.

7. Molin DG, Roest PA, Nordstrand H, Wisse LJ, Poelmann RE, Eriksson UJ and Gittenberger-De Groot AC: Disturbed morphogenesis of cardiac outflow tract and increased rate of aortic arch anomalies in the offspring of diabetic rats. Birth Defects Res A Clin Mol Teratol 70: 927-938, 2004.

8. Dinleyici EC, Tekin N, Dinleyici M, Kilic Z, Adapinar B and Aksit MA: Severe fatal course of axial mesodermal dysplasia spectrum associated with complex cardiac defect in an infant of a mother with insulin dependent diabetes. Am J Med Genet A 15: 2156-2159, 2007

9. Pavlinkova G, Salbaum JM and Kappen C: Maternal diabetes alters transcriptional programs in the developing embryo. BMC Genomics 10: 274, 2009.
10. Jiang B, Kumar SD, Loh WT, Manikandan J, Ling EA, Tay SS and Dheen ST: Global gene expression analysis of cranial neural tubes in embryos of diabetic mice. J Neurosci Res 86: 3481-3493, 2008.

11. Camenisch TD, Molin DG, Person A, Runyan RB, Gittenberger-de Groot AC, McDonald JA and Klewer SE: Temporal and distinct TGFbeta ligand requirements during mouse and avian endocardial cushion morphogenesis. Dev Biol 248: 170-181, 2002.

12. Han M, Yang X, Farrington JE and Muneoka K: Digit regeneration is regulated by Msx1 and BMP4 in fetal mice. Development 130: 5123-5132, 2003.

13. Wang J, Sridurongrit S, Dudas M, Thomas P, Nagy A, Schneider MD, Epstein JA and Kaartinen V: Atrioventricular cushion transformation is mediated by ALK2 in the developing mouse heart. Dev Biol 286: 299-310, 2005.

14. Conway SJ, Henderson DJ and Copp AJ: Pax3 is required for cardiac neural crest migration in the mouse: Evidence from the splotch (Sp2H) mutant. Development 124: 505-514, 1997.

15. Kumar SD, Dheen ST and Tay SS: Maternal diabetes induces congenital heart defects in mice by altering the expression of genes involved in cardiovascular development. Cardiovasc Diabetol 6: 34, 2007.

16. Kumar SD, Yong SK, Dheen ST, Bay BH and Tay SS: Cardiac malformations are associated with altered expression of vascular endothelial growth factor and endothelial nitric oxide synthase genes in embryos of diabetic mice. Exp Biol Med 233: 1421-1432. 2008.

17. Gautier L, Cope L, Bolstad BM and Irizarry RA: Affy-analysis of Affymetrix GeneChip data at the probe level. Bioinformatics 20: 307-315, 2004.

18. Wettenhall JM and Smyth GK: LimmaGUI: A graphical user interface for linear modeling of microarray data. Bioinformatics 20: 3705-3706, 2004.

19. Yu G, Wang LG, Han Y and He QY: Clusterprofiler: An R package for comparing biological themes among gene clusters. OMICS 16: 284-287, 2012.

20. Yu G: Using clusterProfiler to identify and compare functional profiles of gene lists, 2014.

21. Haw R, Hermjakob H, D'Eustachio P and Stein L: Reactome pathway analysis to enrich biological discovery in proteomics data sets. Proteomics 11: 3598-3613, 2011.

22. Fabregat A, Sidiropoulos K, Garapati P, et al: The reactome pathway knowledgebase. Nucleic Acids Research 44: D481-D487, 2015.

23. Kohl M, Wiese S and Warscheid B: Cytoscape: Software for visualization and analysis of biological networks. In: Data Mining in Proteomics, Springer, pp291-303, 2011.

24. van der Linde D, Konings EE, Slager MA, Witsenburg M, Helbing WA, Takkenberg JJ and Roos-Hesselink JW: Birth prevalence of congenital heart disease worldwide: A systematic review and meta-analysis. J Am Coll Cardiol 58: 2241-2247, 2011.

25. Gilboa SM, Correa A, Botto LD, Rasmussen SA, Waller DK, Hobbs CA, Cleves MA and Riehle-Colarusso TJ; National Birth Defects Prevention Study: Association between prepregnancy body mass index and congenital heart defects. Am J Obstet Gynecol 202: 51.e1-51.e10, 2010.

26. Lisowski LA, Verheijen PM, Copel JA, Kleinman CS, Wassink S, Visser GH and Meijboom EJ: Congenital heart disease in pregnancies complicated by maternal diabetes mellitus. An international clinical collaboration, literature review, and meta-analysis. Herz 35: 19-26, 2010.

27. Buraczynska M, Zaluska W, Baranowicz-Gaszczyk I, Buraczynska K, Niemczyk E and Ksiazek A: The intercellular adhesion molecule-1 (ICAM-1) gene polymorphism K469E in end-stage renal disease patients with cardiovascular disease. Hum Immunol 73: 824-828, 2012.

28. Iiyama K, Hajra L, Iiyama M, Li H, DiChiara M, Medoff BD and Cybulsky MI: Patterns of vascular cell adhesion molecule-1 and intercellular adhesion molecule-1 expression in rabbit and mouse atherosclerotic lesions and at sites predisposed to lesion formation. Circ Res 85: 199-207, 1999.

29. Meerschaert J and Furie MB: The adhesion molecules used by monocytes for migration across endothelium include CD11a/CD18, CD11b/CD18 and VLA-4 on monocytes and ICAM-1, VCAM-1, and other ligands on endothelium. J Immunol 154: 4099-4112, 1995.

30. Toyozaki T, Saito T, Takano H, Yorimitsu K, Kobayashi S, Ichikawa $\mathrm{H}$, Takeda $\mathrm{K}$ and Inagaki Y: Expression of intercellular adhesion molecule-1 on cardiac myocytes for myocarditis before and during immunosuppressive therapy. Am J Cardiol 72: 441-444, 1993. 
31. Seko Y, Matsuda H, Kato K, Hashimoto Y, Yagita H, Okumura K and Yazaki Y: Expression of intercellular adhesion molecule-1 in murine hearts with acute myocarditis caused by coxsackievirus B3. J Clin Invest 91: 1327-1336, 1993.

32. Seko Y, Yamazaki T, Shinkai Y, Yagita H, Okumura K, Naito S, Imataka K, Fujii J and Yazaki Y: Cellular and molecular bases for the immunopathology of the myocardial cell damage involved in acute viral myocarditis with special reference to dilated cardiomyopathy. Jpn Circ J 56: 1062-1072, 1992.

33. Donath MY and Shoelson SE: Type 2 diabetes as an inflammatory disease. Nat Rev Immunol 11: 98-107, 2011.

34. Duan M, Yao H, Hu G, Chen X, Lund AK and Buch S: HIV Tat induces expression of ICAM-1 in HUVECs: Implications for miR-221/-222 in HIV-associated cardiomyopathy. PLoS One 8: e60170, 2013.

35. Alvarez D, Briassouli P, Clancy RM, Zavadil J, Reed JH Abellar RG, Halushka M, Fox-Talbot K, Barrat FJ and Buyon JP: A novel role of endothelin-1 in linking Toll-like receptor 7-mediated inflammation to fibrosis in congenital heart block. J Biol Chem 286: 30444-30454, 2011.

36. Yamamoto H, Williams EG, Mouchiroud L, Cantó C, Fan W, Downes M, Héligon C, Barish GD, Desvergne B, Evans RM, et al: NCoR1 is a conserved physiological modulator of muscle mass and oxidative function. Cell 147: 827-839, 2011.

37. Lockhart MM, Wirrig EE, Phelps AL, Ghatnekar AV, Barth JL, Norris RA and Wessels A: Mef2c regulates transcription of the extracellular matrix protein cartilage link protein 1 in the developing murine heart. PLoS One 8: e57073, 2013.

38. Planavila A, Dominguez E, Navarro M, Vinciguerra M, Iglesias R, Giralt M, Lope-Piedrafita S, Ruberte J and Villarroya F: Dilated cardiomyopathy and mitochondrial dysfunction in Sirt1-deficient mice: A role for Sirt1-Mef2 in adult heart. J Mol Cell Cardiol 53: 521-531, 2012.
39. Ventura-Clapier R, Garnier A and Veksler V: Energy metabolism in heart failure. J Physiol 555: 1-13, 2004.

40. Pirinccioglu AG, Alyan O, Kizil G, Kangin M and Beyazit N: Evaluation of oxidative stress in children with congenital heart defects. Pediatr Int 54: 94-98, 2012.

41. Vinciguerra M, Santini MP, Martinez C, Pazienza V, Claycomb WC, Giuliani A and Rosenthal N: mIGF-1/JNK1/SirT1 signaling confers protection against oxidative stress in the heart. Aging Cell 11: 139-149, 2012

42. Alcendor RR, Gao S, Zhai P, Zablocki D, Holle E, Yu X, Tian B, Wagner T, Vatner SF and Sadoshima J: Sirt1 regulates aging and resistance to oxidative stress in the heart. Circ Res 100: 1512-1521, 2007.

43. Mottis A, Mouchiroud L and Auwerx J: Emerging roles of the corepressors NCoR1 and SMRT in homeostasis. Genes Dev 27: 819-835, 2013.

44. Hers I, Vincent EE and Tavaré JM: Akt signalling in health and disease. Cell Signal 23: 1515-1527, 2011.

45. Chang Z, Zhang Q, Feng Q, Xu J, Teng T, Luan Q, Shan C, Hu Y, Hemmings BA, Gao X and Yang Z: Deletion of Aktl causes heart defects and abnormal cardiomyocyte proliferation. Dev Biol 347: 384-391, 2010.

46. Dimopoulos K, Diller GP, Koltsida E, Pijuan-Domenech A Papadopoulou SA, Babu-Narayan SV, Salukhe TV, Piepoli MF, Poole-Wilson PA, Best N, et al: Prevalence, predictors, and prognostic value of renal dysfunction in adults with congenital heart disease. Circulation 117: 2320-2328, 2008.

This work is licensed under a Creative Commons Attribution-NonCommercial-NoDerivatives 4.0 International (CC BY-NC-ND 4.0) License. 